\title{
Diffuse-field sound absorption characteristics of a spherical-microperforated space absorber
}

\author{
Kimihiro Sakagami ${ }^{1, *}$, Midori Kusaka ${ }^{1}$, Takeshi Okuzono ${ }^{1}$, Shigeyuki Kido ${ }^{2}$ and Daichi Yamaguchi ${ }^{2}$ \\ ${ }^{1}$ Environmental Acoustics Laboratory, Department of Architecture, Graduate School of Engineering, Kobe University, \\ Rokko, Nada-ku, Kobe, 657-8501 Japan \\ ${ }^{2}$ NC Industry Co., Ltd., 170-1 Shintamaki, Ichida, Kumiyama-cho, Kuze-gun, Kyoto, 613-0022 Japan
}

(Received 30 January 2020, Accepted for publication 29 February 2020)

Keywords: Sound absorption, Microperforated panel, Spherical space absorber PACS number: 43.55.Dt, 43.55.Ev [doi:10.1250/ast.41.784]

\section{Introduction}

Since the pioneering work by Maa [1], sound absorbers using a microperforated panel (MPP) have been intensively studied [2,3]. MPPs are conventionally used with a rigid-back wall and an air-cavity in-between, and the predicting theory for the conventional type was established in Maa's studies $[1,4]$. The recent development of MPPs leads to sound absorbers of attractive design [5]. However, in most cases MPPs are used in the conventional style on a room interior surface.

In our previous studies [6-9], three-dimensional MPP space absorbers (3D-MPAs) were proposed as an alternative use of conventional MPPs. A 3D-MPA is a three-dimensional object made with an MPP in shapes such as cylinder, rectangular, etc. By using MPPs in this way, it is possible to add necessary sound absorption to a room without changing its interior design, also enables one to use them as a kind of sound absorbing furniture with attractive design. So far, a cylindrical MPP space absorber (CMSA) [6] and a rectangular MPP space absorber (RMSA) [7], and their variants, e.g., with core and porous filling [8,9], were experimentally and theoretically studied.

These previous works show that CMSA and RMSA offer moderate sound absorption with a broad peak at midfrequencies. Also, CMSAs show better sound absorption performance than RMSAs. This was inferred to be caused by the difference in the sound incidence condition on their surfaces: in the case of CMSA the sound is incident normal to the surface, whereas in the case of RMSA the sound is incident with an angle. As an MPP shows higher absorptivity in the normal incidence condition, CMSAs are thus considered to show better absorption performance than RMSAs. Considering this discussion, we assumed that a shape with round surface can offer better performance. This assumption leads to another assumption that a spherical shape could offer better performance than a CMSA.

Not only the improvement of acoustical performance, but 3D-MPAs of different shapes will give further opportunity for developing designability of sound absorbers. For examples, recently $\mathrm{Li}$ et al. [10] studied a possibility of the prediction of the absorption performance of MPP absorbers of arbitrary

*e-mail: saka@kobe-u.ac.jp shapes. Therefore, in order to confirm the above mentioned assumption, we performed an experiment to measure the reverberation sound absorptivity of spherical MPP space absorbers (SMSA). In this letter, the method of trial production of an SMSA and the results of the absorptivity of an SMSA measured in a reverberation chamber are reported.

\section{Experiment}

2.1. Trial production of SMSA

The trial production of SMSA specimens was made by the following procedure. First, a rectangular flat MPP was made out of a polypropylene sheet. The MPP parameters are summarised in Table 1. These parameters are the same as the specimens used in the authors' previous studies on 3DPMAs. We chose them for comparison purpose. Also, these parameters are quite typical as to obtain good absorption performance.

Secondly, the MPP above was cut into the shape as development drawing of a hemisphere shown in Fig. 1. The MPP cut in this shape was then curved into a hemisphere: the leaves were bent by hand with a hemispherical mould. The edges of leaves were thus contacted each other and bonded to form a hemispherical MPP. Two hemispherical MPPs made in this way were bonded at the glued part on the edges $(5 \mathrm{~mm}$ wide). The contacted parts were tightly bonded so that the air tightness of the cavity is kept. The manufacturing process above is graphically represented in Fig. 2. The photograph of the specimen is shown in Fig. 3.

The specimen made by the above manufacturing process is of $0.152 \mathrm{~m}$ radius (i.e., $0.304 \mathrm{~m}$ diameter) and $0.291 \mathrm{~m}^{2}$ surface area. With the specimens in this diameter, the diameter becomes close to that of the specimens used in CMSA experiments [6]. According to the experimental results for CMSAs [6], a clear resonance peak did not appear in the case of CMSA of larger size $\left(2 \mathrm{~m}^{2}\right.$ surface area, and $0.319 \mathrm{~m}$ radius). Therefore, we considered that the size above is appropriate for the present purpose. Also, the surface area becomes close to that of CMSA specimens (radius $0.159 \mathrm{~m}$, surface area $1 \mathrm{~m}^{2}$ ) when compared with three SMSA specimens.

In the manufacturing procedure, the pitch of the perforations became irregular in the parts where the cutting edges 
Table 1 The parameters of MPP used for the trial production of SMSA specimens.

Hole diameter $(\mathrm{mm}) \quad$ Thickness $(\mathrm{mm}) \quad$ Perforation ratio $(\%)$

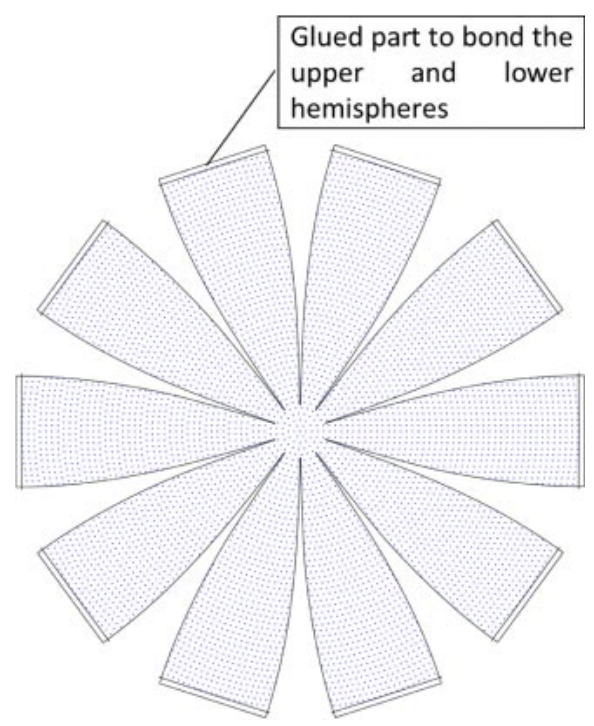

Fig. 1 Development drawing of a hemispherical MPP (the half of an SMSA).

(a)
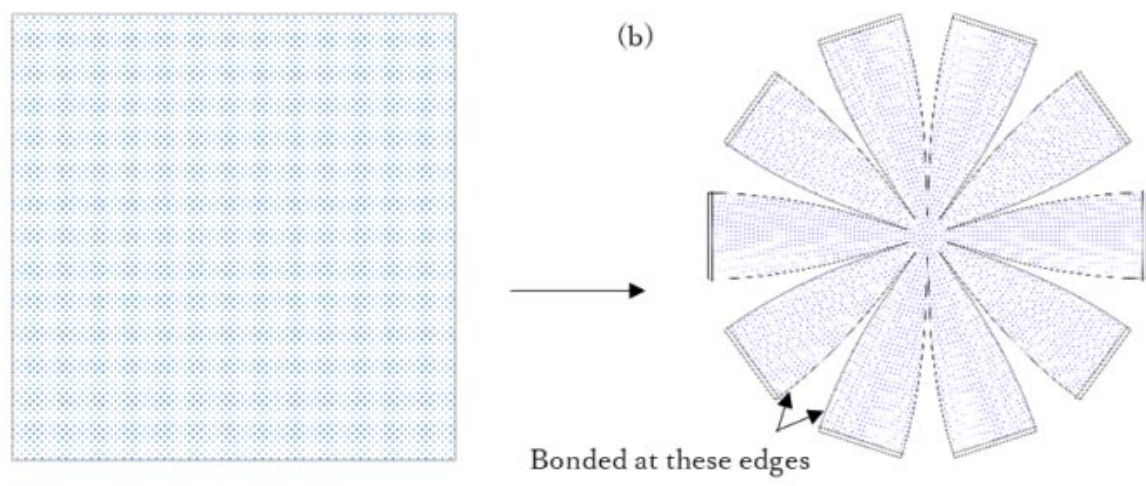

(c)

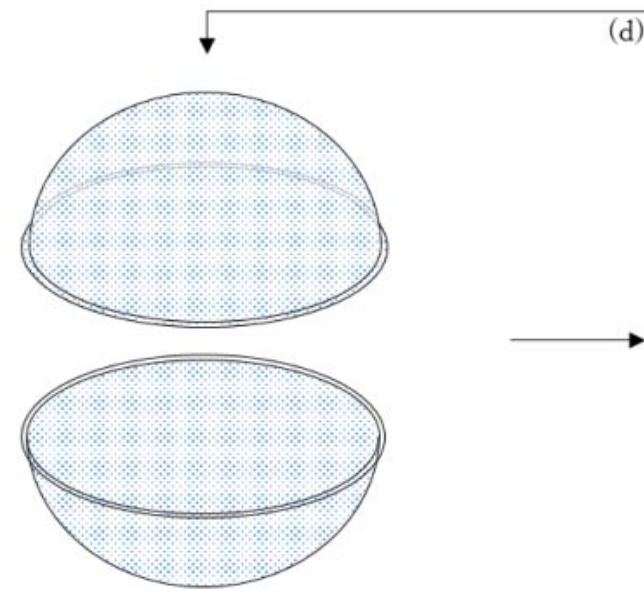

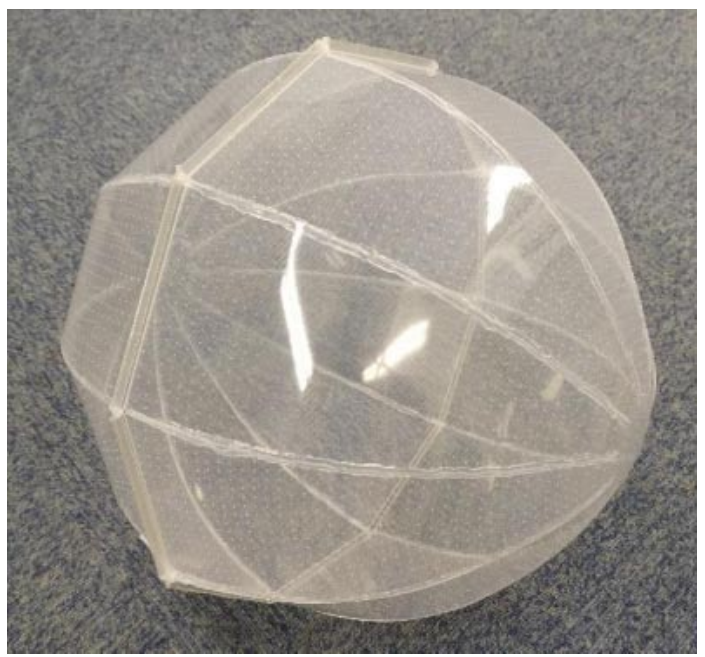

Fig. 3 A photograph of the specimen of an SMSA produced by the above procedure.

are glued, however, they are limited to quite small parts of which the effects are supposed to be negligible for the absorption performance.

\subsection{Measurement}

The measurement of reverberation absorptivity was performed by interrupted noise method according to JIS A 1409 (ISO 354 compatible). Two source positions, and three

(b)

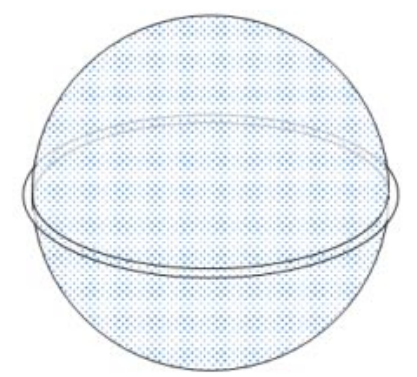

Fig. 2 Diagram of the manufacturing procedure of an SMSA. (a) Prepare a rectangular MPP sheet; (b) Cut (a) into the shape of the development drawing in Fig. 1; (c) Bend the leaves of (a) to form a hemisphere using a mould; (d) two hemispheric MPPs (c) are glued each other with a glued part at the perimeters to form a sphere. 


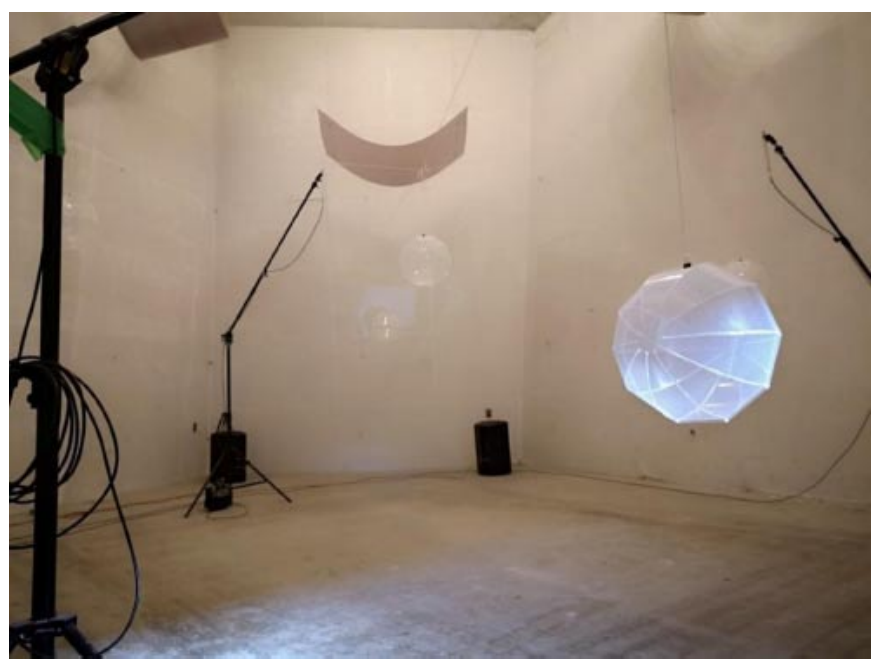

Fig. 4 An example of the experimental set-up and the arrangement of the specimens.

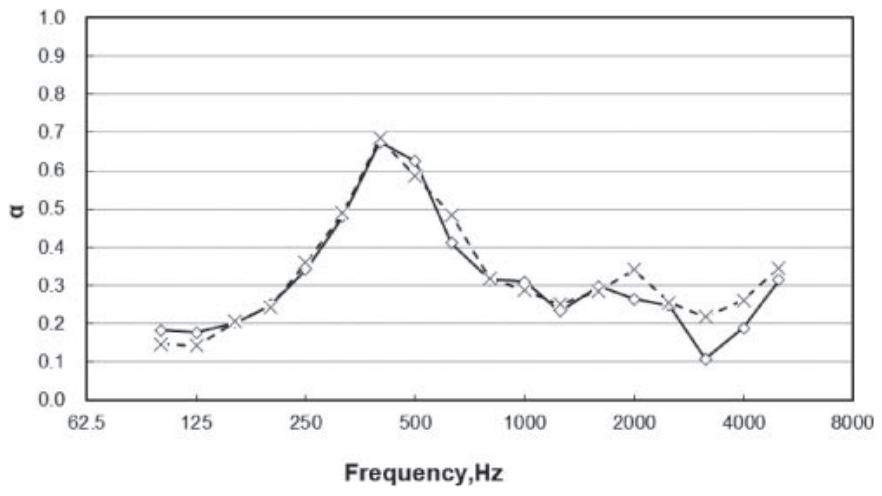

Fig. 5 Comparison of the measured results for the conditions (A) five specimens (solid line), and (B) three specimens (dashed line).

microphone positions were used in the measurement. The volume and surface of the reverberation chamber are $317.4 \mathrm{~m}^{3}$, and $282.3 \mathrm{~m}^{2}$. The test specimens were suspended by wires in the chamber, and placed as far from the walls and each other as possible. In the above condition, the reverberation time $\mathrm{T} 20 \mathrm{~s}$ were measured. The measurements were made in the three conditions: (A) with five specimens; (B) with three specimens; (C) empty condition, including the wires to suspend the specimens. Conditions (A) and (B) were measured for checking whether an area effect occurred, and this point is later discussed. An example of the experimental set up is shown in Fig. 4.

\section{Results and discussion}

First, the effect of the number of the specimen is discussed. As mentioned above, by comparing the results for the conditions (A) and (B), it is confirmed whether an area effect was occurred. The results are presented in Fig. 5. In this figure, the absorptivity is represented as the measured absorption area for one specimen divided by its surface area $\left(0.291 \mathrm{~m}^{2}\right)$, which we call "absorption coefficient" and denote with $\alpha$ in the following discussions.
From the results, the value for one specimen does not change significantly between the conditions (A) and (B). There is a somewhat noticeable difference at $3,150 \mathrm{~Hz}$, however, a difference of this degree is often seen in reverberation chamber measurement, so it can be considered to be within the uncertainty of the measurement. Considering this discussion, it can be concluded that there was no significant area effect and the results derived from five specimens, the condition (A), are used in the following discussion.

As for the absorption performance of SMSAs, the peak absorption at $400 \mathrm{~Hz}$ shows about $\alpha=0.7$ for $1 \mathrm{~m}^{2}$ surface area. The surface area of the present specimen is $0.291 \mathrm{~m}^{2}$, namely, three specimens of SMSA should show approximately this value. This value is higher than the peak values of CMSA and RMSA of $1 \mathrm{~m}^{2}$ surface area [6,7]. Therefore, SMSAs can offer good sound absorption performance, and can be used effectively.

Next, the measured results of SMSAs are compared with those of CMSAs and RMSAs. In the following comparisons, the absorptivity is also compared by comparing measured reverberation absorption per $1 \mathrm{~m}^{2}$ of the surface area. In Refs. [6,7], the measurements were performed for samples of $1 \mathrm{~m}^{2}$ and $2 \mathrm{~m}^{2}$ surface area, but here only $1 \mathrm{~m}^{2}$ results are taken for comparisons. The MPP parameters are the same as in the present study, but the material was different: CMSAs and RMSAs were made of polycarbonate sheets. The diameter of the CMSA is $0.318 \mathrm{~m}$, and the diagonal of the RMSA is $0.35 \mathrm{~m}$, which are close to the diameter of the present SMSA $(0.304 \mathrm{~m})$. As the CMSAs and RMSAs have top openings, the effect of the cover to close the openings were measured. Although, the effect of the cover on the top opening was found to be small, here the comparison is made in the cases both with and without the cover.

The comparison results are shown in Figs. 6 (with CMSAs) and 7 (with RMSAs). As shown in the figures, in both cases, SMSAs show sharper and higher absorption peak at around $400 \mathrm{~Hz}$ which is the same as CMSAs and RMSAs: the peak frequency is considered to be determined mainly by the size of the cavity. The higher peak of SMSAs is inferred to be attributed to their sound incidence condition: in the case of SMSAs the sound from any direction hits the surface along its normal, by which the sound incidence condition becomes close to the normal incidence on its whole surface. Another reason can be that the Helmholtz resonance effect appears more significantly in an SMSA due to its spherical shape. Regarding the effect of the cover on the top openings of CMSAs and RMSAs, when the openings were closed, the absorptivity was only slightly increased [6,7], therefore, the peak absorption of SMSAs was higher in this case as well.

\section{Concluding remarks}

In this letter, the results of a case study of trial production and measurements of the absorption performance of a spherical MPP space absorber (SMSA) were reported. The absorptivity of SMSAs was measured in a reverberation chamber, and found to offer higher sound absorption peak than existing three-dimensional MPP space absorbers, i.e., CMSAs and RMSAs. The results suggest that SMSAs can be 

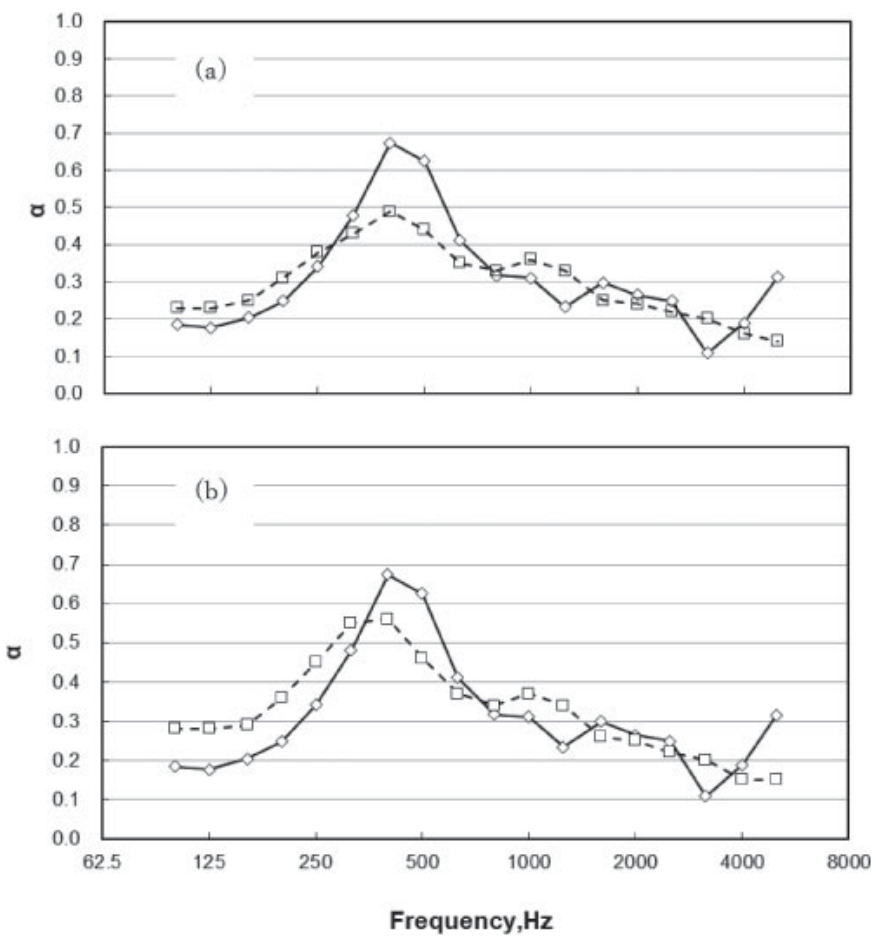

Fig. 6 Comparison of the absorptivity of SMSAs (solid line) and CMSAs (dashed line) without covers (a), and with covers (b), on the top opening.

an alternative as a designable sound absorber. The detailed discussions on the sound absorbing mechanism, theoretical prediction and practical considerations such as cost study, implementation, etc., will be the subjects of a follow-up report.

\section{References}

[1] D.-Y. Maa, "Theory and design on microperforated panel sound-absorbing constructions," Sci. Sin., 18, 55-71 (1975).

[2] D. Herrin, J. Liu and A. Seybert, "Properties and applications of microperforated panels," Sound Vib., 45(7), pp. 6-9 (2011).

[3] D. Herrin, "A guide to the application of microperforated panel absorbers," Sound Vib., 51(12), pp. 12-18 (2017).

[4] D.-Y. Maa, "Microperforated-panel wideband absorbers," Noise Control Eng. J., 29, 77-84 (1987).

[5] T. Adams, Sound Materials: A Compendium of Sound Absorbing Materials for Architectural Design (Frame Publishers, New York, 2017).

[6] K. Sakagami, T. Oshitani, M. Yairi, E. Toyoda and M.
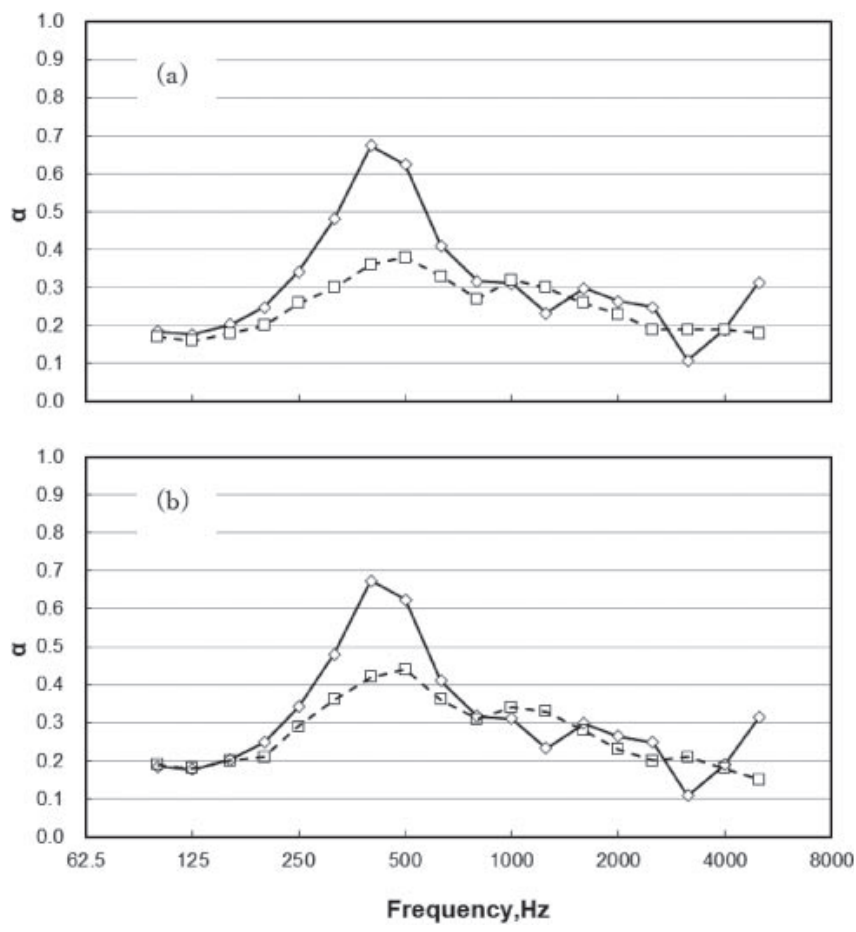

Fig. 7 Comparison of the absorptivity of SMSAs (solid line) and RMSAs (dashed line) without covers (a), and with covers (b), on the top opening.

Morimoto, "An experimental study on a cylindrical microperforated panel space absorber," Noise Control Eng. J., 60, 22-28 (2012).

[7] K. Sakagami, M. Yairi, M. Toyoda and E. Toyoda, "An experimental study on the sound absorption of three-dimensional MPP space sound absorbers: Rectangular MPP space sound absorber (RMSA)," Acoust. Aust., 60, 22-28 (2013).

[8] M. Toyoda, S. Fujita and K. Sakagami, "Numerical analyses of the sound absorption of cylindrical microperforated panel space absorbers with cores," J. Acoust. Soc. Am., 138, 35313538 (2015).

[9] M. Toyoda, K. Sakagami, M. Okano, T. Okuzono and E. Toyoda, "Improved sound absorption performance of threedimensional MPP space sound absorbers by filling with porous materials," Appl. Acoust., 116, 311-316 (2017).

[10] X. Li, Y. Wang, Z. Cai, J. Zhao and X. Gai, "Sound absorption prediction for an arbitrarily shaped microperforated membrane space absorber," Proc. Internoise 2019, Madrid, Spain, June (2019). 